
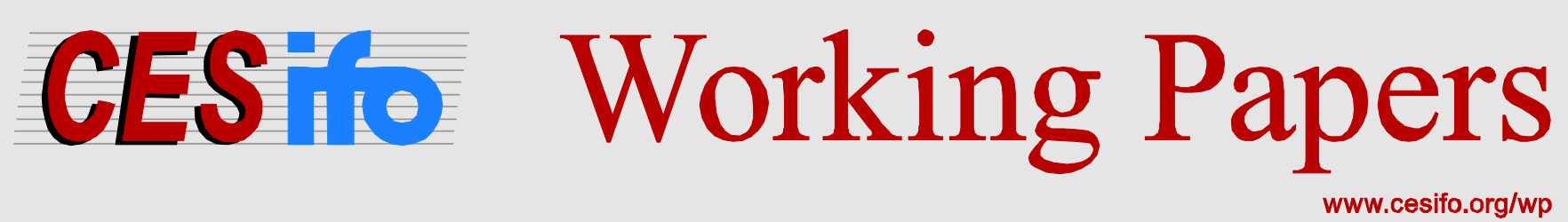

\title{
The Quantitative View of Myerson Regularity
}

\author{
Nikolaus Schweizer \\ Nora Szech
}

\author{
CESIFO WORKING PAPER NO. 5712 \\ CATEGORY 12: EMPIRICAL AND THEORETICAL METHODS \\ JANUARY 2016
}

An electronic version of the paper may be downloaded

- from the SSRN website:

- from the RePEc website:

- from the CESifo website: wWw.SSRN.com

Www.RePEc.org

www.CESifo-group.org/wp 


\title{
The Quantitative View of Myerson Regularity
}

\begin{abstract}
In mechanism design, Myerson regularity is often too weak for a quantitative analysis of performance. For instance, ratios between revenue and welfare, or sales probabilities may vanish at the boundary of Myerson regularity. This paper therefore explores the quantitative version of Myerson regularity, which we call $\lambda$-regularity. It measures how Myerson regular a distribution is. In doing so, we unify separate literatures in economics, computer science, applied mathematics and statistics. The concept has appeared before, e.g., under the names of $\rho$ concavity and $\alpha$-strong-regularity. We provide many new results for quantitative auction and mechanism design.
\end{abstract}

JEL-codes: D440, D470, D820.

Keywords: $\lambda$-regularity, Myerson regularity, monotone hazard rate, auctions, mechanism design, approximation.

\author{
Nikolaus Schweizer \\ Mercator School of Management \\ Duisburg-Essen University \\ Germany-47057 Duisburg \\ nikolaus.schweizer@uni-due.de
}

\author{
Nora Szech \\ Department of Economics \\ Karlsruhe Institute of Technology \\ Germany - 76128 Karlsruhe \\ nora.szech@kit.edu
}

January 2016

We thank Sven Balder, Thomas Mariotti, and Dominik Rothenhäusler. An early version of the manuscript circulated under the title "A Quantitative Version of Myerson Regularity”. 


\section{Introduction}

Since Myerson (1981)'s seminal study of optimal auctions, his regularity condition of increasing virtual valuations has been a cornerstone of the theory of auctions and mechanism design. Yet, Myerson regularity alone is often too weak to guarantee that a model is sufficiently well-behaved for quantitative analysis, such as comparisons of welfare versus revenues, or controlling probabilities of sale in an auction. This liberality of Myerson regularity has become particularly apparent in the recent literature on algorithmic mechanism design, where it typically needs enforcement by additional assumptions. ${ }^{1}$ This literature aims at turning mechanism design more scalable and more quantitative, and therefore better suited for many real world problems. By replacing the classical objective of optimality with the more modest goal of guaranteeing a good approximation of the optimal outcome, various challenging problems (asymmetry, uncertainty about distributions, complex preferences over multiple objects...) can now be handled to a degree that was previously out of reach. For example, several papers analyze successful auction design if only a sample from the bid distribution is known, thereby dropping the classical assumption of common knowledge of distributions. ${ }^{2}$ Thus, these analyses address Wilson (1987)'s critique that asked for a more detail-free approach towards mechanism design.

In many applications, it is desired to estimate quantities such as the ratio of revenue to welfare for a large class of admissible distributions - ideally, for all regular distributions. Yet, as the distributions at the boundary of Myerson regularity behave badly, uniform estimates for all regular distributions cannot exist. In such cases, quantitative estimates have often been obtained by restricting the set of admissible distributions to those that fulfill the monotone hazard rate condition, also known as increasing failure rate (IFR). ${ }^{3}$ Quantitatively, IFR distributions are easier to handle as they have convenient properties such as log-concavity of the survival function " $1-F "$. Further, they easily compare to exponential distributions. This facilitates analysis a lot.

\footnotetext{
${ }^{1}$ See Hartline (2014) and Roughgarden (2015).

${ }^{2}$ See, e.g., Dhangwatnotai et al. (2014) or Azar et al. (2014).

${ }^{3}$ We provide various references in Section 3.1. The monotone hazard rate condition is, of course, not a recent invention but has been a convenient assumption in the microeconomics literature for decades, see e.g. Fudenberg and Tirole (1991).
} 
Moving from the Myerson regular to the monotone hazard rate case, the class of admissible distributions becomes considerably smaller. For example, the class of Myerson regular distributions contains heavy-tailed (power law) distributions. These are ruled out under the monotone hazard rate condition, under which the exponential distribution marks the boundary of the admissible heaviness of tails. Likewise, the monotone hazard rate condition rules out some local irregularities of distributions that are still admissible under Myerson regularity. Both aspects may matter in many applications. Thus, it would be good to impose less than the monotone hazard rate condition, while still being able to provide quantitative results for which Myerson regularity alone is not strong enough.

In this paper, we study an alternative strengthening of Myerson regularity. We define a distribution as $\lambda$-regular if the slope of the virtual valuations function is bounded from below by $\varepsilon=1-\lambda$ for some $\lambda \leq 1$. 1-regularity is thus exactly Myerson regularity. $\lambda=0$, i.e. $\varepsilon=1$, corresponds to the monotone hazard rate condition. Choosing $\varepsilon \in(0,1)$ interpolates between the two cases. This condition has appeared before in the literature on auctions and related topics. It coincides with the $\rho$-concavity assumption of, e.g., Ewerhart (2013) and Mares and Swinkels (2011, 2014) and with the concept of $\alpha$-strong regularity in Cole and Roughgarden (2014), with $\rho=-\lambda$ and $\alpha=1-\varepsilon$.

These authors have different perspectives on the condition which we unify in the present paper. The main contribution of this paper is twofold. First, many of the key tools in the algorithmic mechanism design literature carry over with small changes from the monotone hazard rate to the general $\lambda$-regular case. ${ }^{4}$ Second, $\lambda$-regularity has deep roots in several literatures which implies that it can be exploited in various ways. $\lambda$-regularity has an alternative representation in terms of monotonicity of a generalized hazard rate, a generalized concavity condition on the survival function, and it allows for comparisons (in the convex-transform order) with an explicit class of reference distributions. These properties link $\lambda$-regularity with two rich and distinct bodies of work in applied mathematics, the literature on generalized notions of concavity and the literature on reliability and stochastic orders. ${ }^{5}$ The latter,

\footnotetext{
${ }^{4}$ We would like to point out that a similar project was carried out independently in parallel work by Cole and Rao (2015), see below for more discussion.

${ }^{5}$ For pointers to the former literature, see, e.g., Balogh and Ewerhart (2014), for an introduction
} 
statistical implications have been applied in auction theory mainly for the monotone hazard rate case.

Combining these perspectives and the tools they imply, we first extend a series of quantitative estimates about the single bidder case from the monotone hazard rate case to the $\lambda$-regular case. These results have been used heavily in the algorithmic mechanism design literature. There, quantitative estimates for $\lambda=0$ are often contrasted against their breakdown for $\lambda=1 .{ }^{6}$ Our estimates continue to hold for any $\lambda<1$ with constants that (must) blow up in the limit $\lambda \rightarrow 1$. In particular, we confirm that it is, in a sense, merely the boundary of Myerson regularity that typically causes problems. We then turn to a number of results about order statistics and the $n$-bidder case. We provide a lower bound on the revenue-to-welfare ratio in single-object auctions, and some additional bounds on small order statistics. As an application beyond mechanism design, the latter bounds also provide sharp estimates for a family of generalized Gini indices for the measurement of inequality.

\section{Related Literature}

To our knowledge, so far, the connection between $\lambda$-regularity and results in reliability theory has been explored in economics only for the monotone hazard condition and the comparison with the exponential distribution it entails. In some applications, the monotone hazard rate condition is a boundary case at which the comparative statics of the model change fundamentally. ${ }^{7}$ In other applications, monotone hazard rates are assumed in order to obtain some quantitative control of the underlying distributions. In the latter cases, $\lambda$-regularity is an attractive replacement. There is a small literature investigating the connection between generalized concavity and conditions related to increasing virtual valuations. Applications of generalized concavity in economics begin with Caplin and Nalebuff (1991a,b). Our work is related to applications in equilibrium and price theory such as Anderson and Renault (2003) or Weyl and Fabinger (2013) and, especially, the applications in auction theory by Ewerhart (2013) and Mares and Swinkels (2011, 2014). Of these works,

to the latter Marshall and Olkin (2007) or Shaked and Shanthikumar (2007).

${ }^{6}$ For a typical example, see Aggarwal et al. (2009).

${ }^{7}$ For instance, whether the expected difference between the two highest valuations is increasing or decreasing in the number of bidders switches at the exponential boundary case. This observation is central, e.g., to Moldovanu et al. (2007, 2008) and to Szech (2011). 
Anderson and Renault (2003) are specifically close in spirit to our paper, though they study a different context. They derive elementary, quantitative bounds on key quantities of a Cournot competition model in terms of generalized concavity of the demand (or survival) function. Ewerhart (2013) is complementary to our paper, providing explicit conditions for $\lambda$-regularity in terms of the density function, and a discussion of parametric distribution classes that satisfy the condition. ${ }^{8}$ Moreover, generalized concavity has been applied in the study of procurement auctions with asymmetric valuation distributions by Mares and Swinkels (2011, 2014). In the procurement setting, small realizations of a cost parameter replace large realizations of valuations. Therefore, issues of heavy tails and infinite expected values do not arise like in classical auctions. Mares and Swinkels (2011, 2014) focus on handling two challenging particular applications. Our focus is on providing $\lambda$-regular versions of key tools that have been used in numerous papers.

Finally, Cole and Roughgarden (2014) propose the condition under the name $\alpha$ strong regularity with similar intentions as ours, providing a new tool for algorithmic mechanism design. They identify the family of boundary cases in Definition 1 below and show a bound on selling probabilities, see Lemma 3 below. Independently from us and parallelly, Cole and Rao (2015) have extended the approach of Cole and Roughgarden (2014) to many of the results of our Section 3. Beyond this overlap, Cole and Rao (2015) and our paper are highly complementary. While Cole and Rao develop the implications in algorithmic game theory in far more detail than we do, our work connects the concept to deep results in several earlier literatures in economics and applied mathematics. For instance, the results on $\lambda^{*}$-regularity in Section 4 provide a further generalization of the crucial Lemma 3, relying on a very classical line of reasoning. In addition, the techniques of proof in the two papers are very different. While we interpret $\lambda$-regularity as a property which is (among others) analogous to the usual monotone hazard rate condition from a more abstract perspective, Cole and Rao use $\lambda$-regularity mostly as a more general growth condition on the classical hazard rate.

\footnotetext{
${ }^{8}$ Ewerhart's interest in $\lambda$-regular distributions is not so much motivated by quantitative considerations but rather by the fact that in some generalized auction models the classical regularity condition is replaced by $\lambda$-regularity. See his paper for references. Our results also apply in these settings.
} 
So far, there has been no consensus in the literature concerning the optimal parametrization of this family of conditions. Our parametrization departs both from the literature on $\rho$-concavity $(\rho=-\lambda)$ and from that on $\alpha$-strong regularity $(\alpha=1-\lambda)$. Our parametrization is motivated by two arguments: The three most important special cases are $\lambda=-1, \lambda=0$ and $\lambda=1$, corresponding to comparisons with the uniform, exponential and equal-revenue distribution. Myerson regularity, the classical benchmark, is $\lambda=1$.

The statistical implications of $\lambda$-regularity have hardly been explored beyond the case $\lambda=0$. For one thing, this concerns the direct use of tools from reliability theory. For another, the formulation of $\lambda$-regularity in terms of a generalized monotone hazard rate condition proves to be powerful in several of our proofs since it enables us to directly adapt arguments from the monotone hazard rate case. As all these perspectives are equivalent, it is possible to replace one technique of proof by another in some cases. Yet often, a result follows much more easily from one perspective than from another. ${ }^{9}$

\section{Outline}

Section 2 introduces $\lambda$-regularity, discusses equivalent formulations and provides some structural properties such as existence of moments and invariance under monotone, concave transformations. Section 3 presents our performance bounds and discusses their implications in the algorithmic mechanism design literature. Section 4 shows that some of the key results of Section 3 remain valid when $\lambda$-regularity is replaced by an even weaker notion that we call $\lambda^{*}$-regularity. $\lambda^{*}$-regularity is a generalization of the IFRA (increasing failure rate average) property in the same way that $\lambda$-regularity generalizes the monotone hazard rate condition. Section 5.1 points out converses of our results if the slope of virtual valuations is bounded from above rather than from below. Prominent special cases of this condition are the assumptions of a decreasing density or decreasing failure rate. Section 5.2 discusses $\lambda$-regularity in relation to hyperregularity, an alternative strengthening of Myerson

\footnotetext{
${ }^{9}$ For two concrete examples, Ewerhart (2013)'s sufficient condition for $\lambda$-regularity relies on deep results from the generalized concavity literature. In the monotone hazard rate case, similar implications of log-concavity have been studied by Bagnoli and Bergstrom (2005). In contrast, our result on existence of moments under $\lambda$-regularity is very much in the tradition of reliability theory.
} 
regularity proposed by Kleinberg and Yuan (2013). Hyperregularity corresponds to the increasing generalized failure rate (IGFR) condition of Lariviere and Porteus (2001) which has been studied quite a bit in the revenue management literature. We provide pointers to this literature as well. Finally, Section 6 provides an interpretation of $\lambda$-regularity when the framework is transformed from a price-setting to a quantity-setting problem. For the classical regularity condition, interpretations of this transformation go back to Bulow and Roberts (1989). Section 7 concludes. All proofs are in the appendix.

\section{$2 \lambda$-Regularity}

Throughout, we assume that probability distribution functions $F$ have a continuously differentiable density $f$ that is positive in the interior of the support $(\alpha, \omega)$ of $F$ where $0 \leq \alpha<\omega \leq \infty .{ }^{10}$ We treat $F$ as probability distribution on $\mathbb{R}^{+}$with $f(x)=0$ outside the support, and denote by $X$ a random variable distributed according to $F$. Unless otherwise noted, we use the term increasing in the meaning of weakly increasing, and analogously for decreasing, convex and concave.

We say that $F$ is $\lambda$-regular for some (possibly negative) $\lambda \leq 1$ if the generalized virtual valuations function

$$
v_{\lambda}(x)=\lambda x-\frac{1-F(x)}{f(x)}
$$

is increasing over $(\alpha, \omega)$. This is equivalent to assuming that the slope of the classical virtual valuations function $v_{1}$ is bounded from below by $1-\lambda, v_{1}^{\prime}(x) \geq 1-\lambda$. 1 regularity is thus Myerson's regularity assumption, while 0-regularity corresponds to the classical monotone hazard condition, also known as the increasing failure rate condition (IFR). For $\lambda_{1}<\lambda_{2}, \lambda_{1}$-regularity implies $\lambda_{2}$-regularity. In particular, $\lambda$-regularity for some $\lambda<1$ implies Myerson regularity.

In the following, we argue that $\lambda$-regularity with $\lambda \neq 0$ has many properties in common with the increasing failure rate condition $\lambda=0$. As a first step, we define

\footnotetext{
${ }^{10}$ Assuming positivity is essentially without loss of generality as gaps in the support would lead to a violation of Myerson regularity. We use the smoothness assumption mainly in the proof of Proposition 1 where it could be relaxed with the same techniques as in Lemma 1 of Ewerhart (2013).
} 
the generalized failure rate

$$
r_{\lambda}(x)=\frac{f(x)}{(1-F(x))^{1+\lambda}}
$$

As we will see below, monotonicity of $r_{\lambda}$ is equivalent to monotonicity of $v_{\lambda}$. The following lemma states that any distribution $F$ may be expressed in terms of the rate $r_{\lambda}$.

Lemma 1. For $\lambda \leq 1, \lambda \neq 0$, and $x \geq 0$, we can write

$$
1-F(x)=\Gamma_{\lambda}\left(H_{\lambda}(x)\right)
$$

where the decreasing function $\Gamma_{\lambda}: \mathbb{R}^{+} \rightarrow[0,1]$ is given by

$$
\Gamma_{\lambda}(x)= \begin{cases}0 & \text { when } \lambda<0 \text { and } x>-\frac{1}{\lambda} \\ (1+\lambda x)^{-\frac{1}{\lambda}} & \text { otherwise }\end{cases}
$$

and where

$$
H_{\lambda}(x)=\int_{0}^{x} r_{\lambda}(y) d y
$$

For the classical failure rate with $\lambda=0$, this is a familiar result where the function $\Gamma_{\lambda}$ is replaced by its limit $\Gamma_{0}(x)=\exp (-x)$. Based on the lemma, it is straightforward to define families of distributions for which the rate $r_{\lambda}$ is constant. For later use, we define two such families, $G_{\rho, \lambda}$ and $F_{\mu, \lambda}$. These are the same distributions, once parametrized by their constant generalized failure rate and once parametrized by their mean (if it exists). ${ }^{11}$

\section{Definition 1.}

(i) For $\rho>0$ and $\lambda \neq 0$, define the probability distribution $G_{\rho, \lambda}$ by

$$
G_{\rho, \lambda}(x)=1-\Gamma_{\lambda}(\rho \cdot x)=1-(1+\lambda \rho x)^{-\frac{1}{\lambda}}
$$

with support $(0, \infty)$ for $\lambda>0$ and support $\left(0,-\frac{1}{\rho \lambda}\right)$ for $\lambda<0$. The generalized failure rate of $G_{\rho, \lambda}$ satisfies $r_{\lambda}(x)=\rho$ for all $x$ in the support. Moreover, for $\lambda<1$, $G_{\rho, \lambda}$ has mean $\mu=\frac{1}{\rho(1-\lambda)}$.

\footnotetext{
${ }^{11}$ In the definition, the expressions for the mean follow from applying Lemma 7 in the Appendix with $a=0$ and $b=\rho$.
} 
(ii) For $\mu>0, \lambda<1, \lambda \neq 0$, define the probability distribution $F_{\mu, \lambda}$ by

$$
F_{\mu, \lambda}(x)=1-\Gamma_{\lambda}\left(\frac{x}{\mu(1-\lambda)}\right)
$$

with support $(0, \infty)$ for $\lambda \in(0,1)$ and support $\left(0,-\frac{\mu(1-\lambda)}{\lambda}\right)$ for $\lambda<0$. $F_{\mu, \lambda}$ has constant rate $r_{\lambda}(x)=\frac{1}{\mu(1-\lambda)}$ and mean $\mu$.

For $\lambda<0$, the distributions $G_{\rho, \lambda}$ and $F_{\mu, \lambda}$ are thus a subclass of (rescaled) Beta distributions, while the distributions for $\lambda>0$ are Pareto distributions. The distribution $G_{1,1}$ is a horizontal shift of the equal-revenue distribution. The latter is a well-known distribution at the boundary of Myerson regularity. ${ }^{12}$ For $\lambda \rightarrow 0$, $F_{\mu, \lambda}$ converges to an exponential distribution with rate $\mu$. Finally, $\lambda=-1$ yields a uniform distribution.

The next proposition draws the connection between generalized virtual valuations, generalized failure rates, generalized concavity of $1-F$, and a comparison with the boundary cases $G_{\rho, \lambda}$ in the convex-transform order.

Proposition 1. The following claims are equivalent for any $\lambda \neq 0$.

(i) $F$ is $\lambda$-regular, i.e., $v_{\lambda}$ is increasing.

(ii) The rate $r_{\lambda}$ associated with $F$ is increasing.

(iii) The function $1-F$ is $(-\lambda)$-concave, i.e.,

$$
-\frac{(1-F(x))^{-\lambda}}{\lambda}
$$

is concave in $x$.

(iv) $F$ is dominated by $G_{1, \lambda}$ in the convex-transform order, i.e., $H_{\lambda}(x)=G_{1, \lambda}^{-1}(F(x))$ is convex in $x$.

Part (iii) of the proposition corresponds to log-concavity of $1-F$ in the monotone hazard rate case $\lambda=0$, and to concavity of $1-F$ for $\lambda=-1$. The expression "( $-\lambda)$-concavity of $1-F$ " follows the terminology of $\rho$-concavity as applied, e.g., in Ewerhart (2013).

\footnotetext{
${ }^{12}$ See, e.g., the monograph Hartline (2014). The equal-revenue distribution has the distribution function $G_{1,1}(x-1)=1-x^{-1}$ with support $(1, \infty)$.
} 
Many of the ingredients of the proposition have appeared before, in different lines of research. For the regular case $\lambda=1$, the equivalence of (i) and (iii) was first observed in McAfee and McMillan (1987). The equivalence of Myerson's regularity to monotonicity of the rate $r_{1}$ is discussed in Ewerhart (2013) and applied in Szech (2011). In the revenue management literature, parts (i-iii) of the proposition (with $\lambda=1$ ) had independently been suggested as conditions on demand curves. ${ }^{13}$ Equivalence of (iii) and (iv) was observed already in van Zwet (1964, Section 4.3.2). Equivalence of (ii) and (iv) has been applied in the auctions literature mainly for $\lambda=0$ where it corresponds to a comparison with the exponential distribution under the monotone hazard rate condition. ${ }^{14}$ Ewerhart (2013) and Mares and Swinkels (2011, 2014) discuss the family of conditions for varying $\lambda$, focusing mainly on the equivalence of (i) and (iii). ${ }^{15}$ Cole and Roughgarden (2014) and Cole and Rao (2015) mostly apply (i) directly or interpret it as a growth condition on the classical hazard rate $r_{1}$.

Our formulation emphasizes two aspects that will be fruitful to exploit:

(i) Combining the proposition with Lemma 1 enables us to apply monotonicity of the generalized rate $r_{\lambda}$ almost like a classical monotone hazard rate assumption. This idea is a key ingredient in many of the proofs of the following section.

(ii) Part (iv) of the proposition connects $\lambda$-regularity to a sizable literature in reliability theory that considers classes of distributions that are dominated by a reference distribution in terms of the convex-transform order. ${ }^{16}$ The next proposition collects two more structural results that rely on arguments adapted from this literature.

\footnotetext{
${ }^{13}$ See Section 5.2 below and Ziya et al. (2004) for details.

${ }^{14}$ Many applications of the monotone hazard rate condition in auction theory can be interpreted in this way, see the next section for examples. Similarly, the assumption that the density is monotonic, $\lambda=-1$, can often be interpreted as a comparison with the uniform distribution.

${ }^{15}$ Mares and Swinkels $(2011,2014)$ consider procurement auctions where virtual valuations are replaced by virtual costs $x+F(x) / f(x)$ and the focus is on small values of $x$. Since the support of $F$ is bounded from below but not from above, issues of heavy tails and infinite expected values are negligible in the procurement setting.

${ }^{16}$ Two seminal early references are van Zwet (1964) and Barlow and Proschan (1966). See Chapter 4.B of Shaked and Shanthikumar (2007) for an overview and a collection of implications.
} 


\section{Proposition 2.}

(i) Let $F$ be $\lambda$-regular for some $\lambda \in(0,1)$. Then $E\left[X^{p}\right]$ is finite for all $p \in\left[1, \frac{1}{\lambda}\right)$.

(ii) Let $F$ be $\lambda$-regular for some $\lambda \leq 1$ and let the mapping $h: \mathbb{R}^{+} \rightarrow \mathbb{R}^{+}$be concave and strictly increasing. Then the distribution of $h(X)$ is $\lambda$-regular as well.

The first part of the proposition guarantees that $\lambda$-regular distributions with $\lambda<1$ always possess a finite mean. Similarly, all distributions that are $\lambda$-regular for some $\lambda<\frac{1}{2}$ possess a finite variance. ${ }^{17}$ As can be seen from the proof, it actually suffices for $\lambda$-regularity to hold in the tails of a distribution, i.e., from some threshold on. The second part shows that $\lambda$-regularity is inherited by linear shifts and stretches of $F$.

Conversely to $\lambda$-regularity, we define $\lambda$-antiregularity as follows. A distribution $F$ is $\lambda$-antiregular for some $\lambda \leq 1$ if the function $v_{\lambda}(x)$ is decreasing in $x$. While $\lambda$-regularity postulates that the tails of the distribution are not too heavy, $\lambda$ antiregularity postulates that the tails are sufficiently heavy. The most common assumptions of this type are 0-antiregularity which is the classical decreasing failure rate condition (DFR), and (-1)-antiregularity which is equivalent to the density $f$ being decreasing. Many of our results have counterparts in the antiregular case. These are discussed in Section 5.1 below.

\section{Performance Bounds}

This section shows how to generalize performance bounds for sales mechanisms from the monotone hazard rate case to the general $\lambda$-regular case with $\lambda<1$. We thus demonstrate that these performance bounds continue to hold (with different constants) right up to the boundary of Myerson regularity. In typical applications from algorithmic mechanism design, the monotone hazard rate condition enters through elementary observations about the single-bidder case. These observations are used to analyze more complex mechanisms in a modular fashion. For example, Lemma 3 below provides a lower bound on the probability that a single bidder has a valuation

\footnotetext{
${ }^{17}$ In Proposition 2 (ii), we exclude the cases $\lambda \leq 0$. 0-regularity, i.e., the monotone hazard rate condition, implies finiteness of all moments, see Barlow and Proschan (1965). $\lambda$-regularity with negative $\lambda$ implies 0 -regularity and thus existence of all moments.
} 
above Myerson's optimal reserve price. This result implies bounds on the probability that the item is (not) sold even with many bidders. For this reason, most of the results below focus on the single bidder case - even though the intended applications are complex problems with multiple items and many bidders. In particular, how to proceed from the single-bidder case to more complex settings is known in many cases. Generalizing the "input" results for the single-bidder case to $\lambda$-regularity is often sufficient for generalizing the final results. Section 3.1 gathers the key inputs of this type for various papers in the algorithmic mechanism design literature. Some implications of $\lambda$-regularity that explicitly address the case of multiple agents are presented in Section 3.2.

\subsection{Basic Bounds}

When selling to a buyer whose valuation $X$ is distributed according to $F$, the optimal posted price can be found via the virtual valuation function. This is shown in Myerson (1981). The revenue-optimal posted price $p^{*}$ is exactly the value at which the virtual valuation function $v_{1}$ turns zero. The same value $p^{*}$ is also the revenueoptimal reserve price in an auction with many bidders with independent private valuations.

On an unbounded state space, Myerson regularity does not guarantee the existence of the reserve $p^{*}$. $\lambda$-regularity, however, implies existence and uniqueness of $p^{*}$.

Lemma 2. Let $F$ be $\lambda$-regular for some $\lambda<1$. Then there exists a unique reserve $p^{*} \in(\alpha, \omega)$ with $v_{1}\left(p^{*}\right)=0$.

Lemma 3 provides lower bounds on the probabilities that the buyer valuation $X$ lies above the mean of $F$, and above the reserve $p^{*}$, respectively. Both bounds are sharp for boundary cases in which $v_{\lambda}$ is constant.

Lemma 3. Let $F$ be $\lambda$-regular for some $\lambda<1, \lambda \neq 0$. Denote by $\mu$ the mean of $F$ and by $p^{*}$ the unique zero of $v_{1}$, i.e., the reserve. Then the following inequalities hold:

$$
P(X>\mu) \geq \Gamma_{\lambda}\left(\frac{1}{1-\lambda}\right)=(1-\lambda)^{\frac{1}{\lambda}}
$$

and

$$
P\left(X>p^{*}\right)=P\left(v_{1}(X)>0\right) \geq(1-\lambda)^{\frac{1}{\lambda}} .
$$


In the monotone hazard rate case, $\lambda=0$, the constant on the right hand side of both inequalities of Lemma 3 converges to $\exp (-1) \approx 0.368$. In this form, both inequalities have been applied frequently in the literature. In some cases, this is the only place where the monotone hazard rate assumption enters so that the results of the papers generalize instantly to the $\lambda$-regular case. ${ }^{18}$ The dependence of the lower bound on $\lambda$ is shown in Figure 1. We see that $\lambda=0$ is far from an exceptional value in this perspective. At $\lambda=1 / 2$, the threshold where variances may become infinite, the bound is still at 0.25 and thus far from zero.

With Lemma 3 we revisit a well-known result. The first bound can be deduced directly from classical results in reliability theory, using our Proposition 1 (iv). ${ }^{19}$ We present a new proof within our framework as most of the arguments reappear later on. For instance, the generalization of the second bound to the $\lambda^{*}$-regular case in Section 4 relies on analogous reasoning.

As a consequence of Lemma 3, we obtain bounds which control the entire distribution function $F$ up to, respectively, $p^{*}$ and $\mu$ in terms of the boundary cases $F_{\mu, \lambda}$ from Definition 1. In the monotone hazard rate case, a result of this type is the main ingredient of Aggarwal et al. (2009) who study the welfare losses of revenue-optimal mechanisms.

Corollary 1. Let $F$ be $\lambda$-regular for some $\lambda<1, \lambda \neq 0$. Denote by $\mu$ the mean of $F$ and by $p^{*}$ the unique zero of $v_{1}$, i.e., the reserve. Then the following bounds are obtained:

$$
1-F(x) \geq 1-F_{\mu, \lambda}(x)
$$

for $x \in[0, \mu]$, and

$$
1-F(x) \geq 1-F_{p^{*}, \lambda}(x)
$$

for $x \in\left[0, p^{*}\right]$.

The lemma is again sharp for $F=F_{\mu, \lambda}$. For the boundary cases $F_{\mu, \lambda}$, the optimal

\footnotetext{
${ }^{18}$ The first inequality is e.g. used in Hartline et al. (2008) and Azar et al. (2013), the second one in Hartline et al. (2008), Aggarwal et al. (2009), Daskalakis and Pierrakos (2011) and Cigler et al. (2014). In Daskalakis and Pierrakos (2011) and in Cigler et al. (2014), this is the only application of the monotone hazard rate assumption.

${ }^{19}$ The same is true for the first bound in Corollary 1. The second bound is shown in Cole and Roughgarden (2014). See, for instance, Proposition 6.2 in Chapter 4 of Barlow and Proschan (1981).
} 
reserve price $p^{*}$ coincides with the mean $\mu$. This holds as the virtual valuations functions $v_{1}$ are linear if $v_{\lambda}$ is constant. Therefore, ${ }^{20}$

$$
0=E\left[v_{1}(X)\right]=v_{1}(\mu) .
$$

This explains why we obtain the same constants in the two inequalities of Lemma 3. For general $\lambda$-regular distributions, the ranking of $p^{*}$ and $\mu$ is ambiguous. ${ }^{21}$ It depends on the distribution which of the two bounds in Lemma 3 and Corollary 1 are sharper.

The next lemma provides a sharp bound on the probability that a valuation lies above the optimal monopoly profit. This result is a central ingredient in Sundararajan and Yan (2015)'s analysis of robust mechanism design for risk-averse sellers. Sundararajan and Yan (2015) prove the result separately for $\lambda=0$ and $\lambda=1$. $^{22}$

Lemma 4. Let $F$ be $\lambda$-regular for some $\lambda<1, \lambda \neq 0$. Denote by $p^{*}$ the unique zero of $v_{1}$ and by $R^{*}=p^{*}\left(1-F\left(p^{*}\right)\right)$ the optimal monopoly profit. Then the following inequality holds:

$$
1-F\left(R^{*}\right) \geq \Gamma_{\lambda}\left(\frac{\Gamma_{\lambda}\left(\frac{1}{1-\lambda}\right)}{1-\lambda}\right) .
$$

For $\lambda \in\{0,1\}$, the constant on the right hand side converges to the values $1 / 2$ for $\lambda=1$ and $\exp (-\exp (-1)) \approx 0.6922$ for $\lambda=0$ as identified by Sundararajan and Yan (2015). ${ }^{23}$ The (weak) dependence of the lower bound on $\lambda$ is shown in Figure 1 .

Our next result provides a sharp bound between the expected value of $F$ and the supremum of $p(1-F(p))$. When selling to a single buyer, these two quantities can be interpreted as the expected welfare generated by a welfare-optimal mechanism and the expected revenue of a revenue-optimal mechanism. In the terminology of

\footnotetext{
${ }^{20}$ The first equality is an elementary property of virtual valuations which holds whenever $E[X]$ is finite.

${ }^{21}$ One can obtain a clear ranking when virtual valuations are concave or convex by applying Jensen's inequality to $0=E\left[v_{1}(X)\right]$ and using the monotonicity of $v_{1}$.

${ }^{22}$ See their Lemmas 18 and 19. They use different techniques of proof for the two results. Our proof extends their proof for $\lambda=0$.

${ }^{23}$ Unlike most results in this section, this bound does not deteriorate at $\lambda=1$.
} 


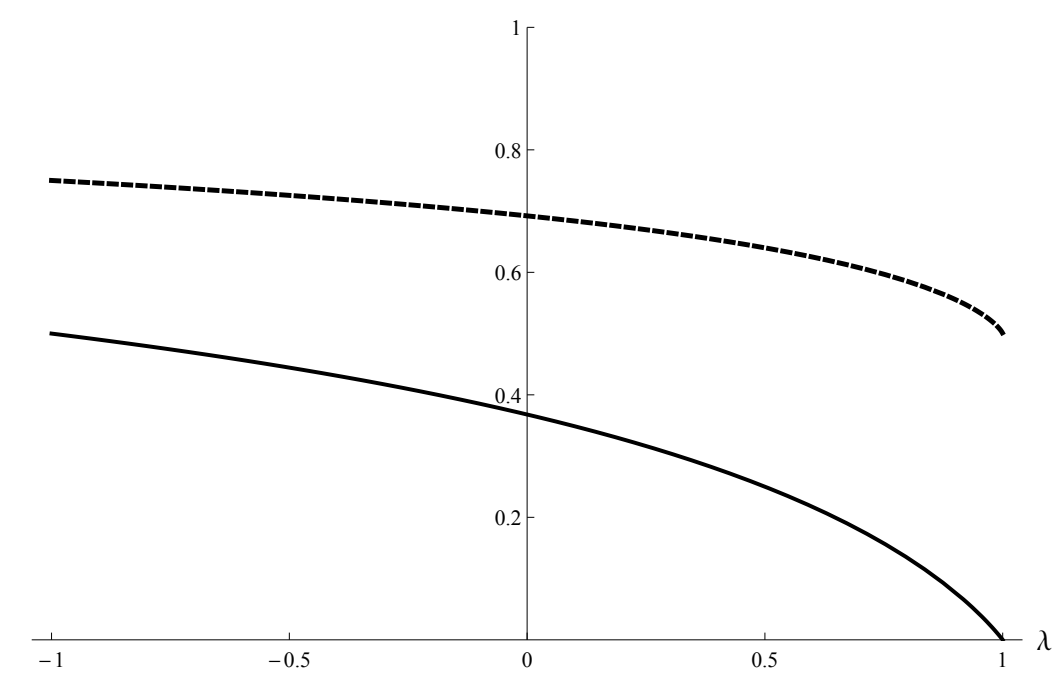

Figure 1: The lower bounds of Lemma 3 (solid line) and Lemma 4 (dashed line) as functions of $\lambda$.

Kleinberg and Yuan (2013), the result shows that $\lambda$-regular distributions are always $c$-bounded with an explicit value of the constant $c .^{24}$

Proposition 3. Let $F$ be $\lambda$-regular for some $\lambda<1, \lambda \neq 0$. Denote by $\mu$ the mean of $F$ and by $p^{*}$ the unique zero of $v_{1}$. Then we have the bound

$$
\mu \leq\left(\frac{1}{1-\lambda}\right)^{\frac{1}{\lambda}} \sup _{p} p(1-F(p))=\left(\frac{1}{1-\lambda}\right)^{\frac{1}{\lambda}} p^{*}\left(1-F\left(p^{*}\right)\right)
$$

Proposition 3 can be seen as a corollary of Proposition 5 in Anderson and Renault (2003) where the result is phrased in the terminology of demand functions and Cournot competition. ${ }^{25}$ A second result in this vein bounds the expected welfare generated by selling with a given reserve price $p$ against expected revenues. The result also applies to suboptimal values of $p$ and can thus be applied in settings where only an estimate of $p^{*}$ is available.

Proposition 4. Let $F$ be $\lambda$-regular for some $\lambda<1, \lambda \neq 0$. Denote by $\mu$ the mean

\footnotetext{
${ }^{24}$ Kleinberg and Yuan (2013) introduce $c$-boundedness as a third, separate assumption in addition to 1-regularity and the hyperregularity condition discussed in Section 5.2. Proposition 3 provides an elementary sufficient condition for $c$-boundedness.

${ }^{25}$ Anderson and Renault (2003) prove several related results but this is the only actual overlap. The techniques of proof are also different since Anderson and Renault rely almost exclusively on the $(-\lambda)$-concavity formulation of $\lambda$-regularity.
} 
of $F$ and by $p^{*}$ the unique zero of $v_{1}$. Then we have the bounds

$$
E\left[X 1_{\{X \geq p\}}\right] \leq\left(\frac{1}{1-\lambda}\right)^{\frac{1}{\lambda}} p^{*}\left(1-F\left(p^{*}\right)\right)
$$

for all $p \geq 0$, and, for $p \geq p^{*}$,

$$
E\left[X 1_{\{X \geq p\}}\right] \leq\left(1+\frac{1}{1-\lambda}\right) p(1-F(p))
$$

In the monotone hazard rate case, this result is a key ingredient in Dhangwatnotai et al. (2014), see also Yan (2012). ${ }^{26}$ The proposition is the only consequence of the monotone hazard condition used in Azar et al. (2014). The latter paper studies sales mechanisms when only a sample from the bid distributions is available while the underlying distributions of valuations are unknown. The authors provide two sets of results, one for the regular case under a symmetry assumption on the bidders, another under the monotone hazard rate condition without the symmetry assumption. Proposition 4 extends the second set of results to the asymmetric, $\lambda$-regular case.

\subsection{Bounds on Order Statistics}

In this section, we show how order statistics can be handled using $\lambda$-regularity. Order statistics frequently arise in auction settings but not only there. At the end of the section, we show that $\lambda$-regularity also implies sharp upper bounds on Gini indices and related quantities.

There are $n$ independent random variables $X_{1}, \ldots, X_{n}$ with distribution $F$. We denote by $X_{k: n}$ the $k^{\text {th }}$ largest of these, so that $X_{1: n}$ is the largest and $X_{n: n}$ is the smallest. ${ }^{27}$ The following lemma states a direct consequence of $\lambda$-regularity:

Lemma 5. Let $F$ be $\lambda$-regular for some $\lambda<1$. Then, the sequence $\left(a_{n}\right)_{n \geq 1}$ defined

\footnotetext{
${ }^{26}$ Dhangwatnotai et al. (2014) combine the two inequalities into a single one with the worse constant $\max (2, \exp (1))=\exp (1)$ for $\lambda=0$. Since the dependence on $\lambda$ differs between the two cases, we keep two separate inequalities.

${ }^{27}$ In particular, we follow the standard notation in auction theory rather than in statistics where $X_{n: n}$ would denote the largest order statistic.
} 
by $a_{1}=(\lambda-1) E\left[X_{1}\right]$ and, for $n \geq 2$,

$$
a_{n}=E\left[X_{2: n}\right]-(1-\lambda) E\left[X_{1: n}\right]
$$

is increasing and concave.

An immediate corollary is the following lower bound on the ratio of optimal revenues and optimal welfare when selling a single asset to $n$ bidders with i.i.d $\lambda$-regular valuations. Recall that with $n$ bidders optimal revenue is given by

$$
R_{n}^{*}=E\left[\max \left(0, v_{1}\left(X_{1: n}\right)\right)\right]
$$

as shown in Myerson (1981). The welfare generated by a welfare-maximizing mechanism is given by $E\left[X_{1: n}\right] \geq R_{n}^{*}$.

Corollary 2. Let $F$ be $\lambda$-regular for some $\lambda<1$. Then we have, for $n \geq 2$, the bound

$$
\frac{R_{n}^{*}}{E\left[X_{1: n}\right]} \geq \frac{E\left[X_{2: n}\right]}{E\left[X_{1: n}\right]} \geq(1-\lambda)\left(1-\frac{E\left[X_{1}\right]}{E\left[X_{1: n}\right]}\right) .
$$

In the corollary, the second inequality bounding second order statistics from below is sharp. We see that $\lambda$-regularity with $\lambda<1$ keeps the ratios on the left hand sides bounded away from zero as $n$ grows. Kleinberg and Yuan (2013) demonstrate by a counterexample ${ }^{28}$ that the same revenue to welfare ratios may vanish for 1-regular distributions and introduce an additional assumption, hyperregularity, to ensure that it remains bounded away from zero. The corollary shows that $\lambda$-regularity rules out counterexamples like this. We further discuss the relation between hyperregularity and $\lambda$-regularity in Section 5.2.

Our final results study the behavior of smallest rather than largest order statistics. We first show an upper bound on the larger out of two independent draws from $F$ in terms of the smaller.

Lemma 6. Let $F$ be $\lambda$-regular for some $\lambda<1$. Then we have the chain of inequalities

$$
E\left[X_{1: 2}\right] \leq \frac{3-\lambda}{2-\lambda} E\left[X_{1}\right] \leq \frac{2-\lambda}{1-\lambda} E\left[X_{2: 2}\right]
$$

\footnotetext{
${ }^{28}$ Roughly speaking, their example differs from boundary cases of 1-regularity only by a logarithmic factor. The example is thus 1-regular but not $\lambda$-regular for any $\lambda<1$.
} 
Again, this result is sharp for boundary cases of $\lambda$-regularity. As $\lambda$ increases, the constants in the chain of inequalities become larger, allowing for a greater spread between the smaller and larger out of two draws. A variation of the argument behind the lemma implies an upper bound on the generalized Gini indices of Donaldson and Weymark (1983), see Kleiber and Kotz (2003) for background.

Corollary 3. Let $F$ be $\lambda$-regular for some $\lambda<1$ and let $n \geq 2$. Then we have the bound

$$
\mathcal{G}_{n}=1-\frac{E\left[X_{n: n}\right]}{E\left[X_{1}\right]} \geq \frac{n-1}{n-\lambda} .
$$

Here, $\mathcal{G}_{2}$ coincides with the classical Gini index which measures economic inequality by comparing the wealth of the poorer of two people to the average wealth in the population. The generalized index $\mathcal{G}_{n}$ replaces the poorest out of two with the poorest out of $n$ people, thus shifting attention to the extremes of the distribution. Increasing $\lambda$ increases the upper bound on economic inequality that is possible under a $\lambda$-regular distribution of wealth. Again, the bound is sharp for all $n$ at the boundary cases of $\lambda$-regularity. ${ }^{29}$

\section{$4 \quad \lambda^{*}$-regularity}

In this section, we show that some of our results already hold under a weaker notion of regularity than $\lambda$-regularity. This includes, in particular the bounds on sales probabilities from Lemma 3 which had been applied frequently for the case $\lambda=0$. The reliability literature (e.g. Barlow and Proschan, 1981) has explored various weaker forms of the monotone hazard rate condition which retain the exponential distribution as a boundary case but place less rigid local restrictions on the shape of the hazard rate. One prominent example is the increasing failure rate average (IFRA) condition. When moving beyond the exponential distribution as a reference distribution, IFRA distributions turn into the class of distributions which are dominated by a reference distribution in terms of the star order ${ }^{30}$ (rather than the stronger convex-transform order we had above). The following proposition defines

\footnotetext{
${ }^{29}$ Donaldson and Weymark (1983) actually define $\mathcal{G}_{n}$ with a continuous parameter $n$. Our result also applies to this case but the term $E\left[X_{n: n}\right]$ in the definition needs to be replaced by it continuous analogue, the right hand side of equation (7) below.

${ }^{30}$ See Shaked and Shanthikumar (2007) for an introduction and various implications.
} 
$\lambda^{*}$-regularity, the analogous weakening of $\lambda$-regularity, and introduces some equivalent formulations:

Proposition 5. The following claims are equivalent for any $\lambda \neq 0$ and $x>0$ :

(i) F is $\lambda^{*}$-regular, i.e., $\frac{H_{\lambda}(x)}{x}$ is increasing in $x$.

(ii) For all $x$,

$$
H_{\lambda}(x) \leq r_{\lambda}(x) \cdot x
$$

(iii) For all $x$ and $\theta \in[0,1]$,

$$
H_{\lambda}(\theta \cdot x) \leq \theta \cdot H_{\lambda}(x)
$$

Since $H_{\lambda}(x)=G_{1, \lambda}^{-1}(F(x)), \lambda^{*}$-regularity is the same as $F$ being dominated by $G_{1, \lambda}$ in the star order which is defined as monotonicity of $G_{1, \lambda}^{-1}(F(x)) / x$. From part (i) of the proposition, we see that at the boundary of $\lambda^{*}$-regularity the function $H_{\lambda}$ is linear with $H_{\lambda}(0)=0 . \quad \lambda^{*}$-regularity thus has the same boundary cases as $\lambda$ regularity. Parts (ii) and (iii) of the proposition always hold when $H_{\lambda}$ is convex. Thus, $\lambda^{*}$-regularity is implied by $\lambda$-regularity. To understand the difference between $\lambda$-regularity and $\lambda^{*}$-regularity better, we write part (iii) of the proposition as

$$
H_{\lambda}(\theta \cdot x+(1-\theta) \cdot 0) \leq \theta \cdot H(x)+(1-\theta) H(0) .
$$

Instead of assuming convexity - and thus global validity of Jensen's inequality $\lambda^{*}$-regularity merely requires that Jensen's inequality holds when one of the points is zero. Another way to see that $\lambda^{*}$-regularity is connected to "convexity in reference to zero" is to write (ii) as

$$
H_{\lambda}(x)-H_{\lambda}^{\prime}(x) \cdot x \leq H_{\lambda}(0)=0 .
$$

Thus, the tangent to $H_{\lambda}$ at a point $x$ need not lie below the graph of $H_{\lambda}$ globally, but it must do so at zero. Looking through Section 3, we see that some of the proofs remain valid under $\lambda^{*}$-regularity since convexity in reference to zero is the only consequence of $\lambda$-regularity we use. 
Corollary 4. Let $F$ be $\lambda^{*}$-regular for some $\lambda<1, \lambda \neq 0$, and suppose there exists a unique $p^{*}$ with $v_{1}\left(p^{*}\right)=0$. Then we have the bounds

$$
P\left(X>p^{*}\right) \geq(1-\lambda)^{\frac{1}{\lambda}}
$$

for $x \in\left[0, p^{*}\right]$

$$
1-F(x) \geq 1-F_{p^{*}, \lambda}(x),
$$

and

$$
1-F\left(R^{*}\right) \geq \Gamma_{\lambda}\left(\frac{\Gamma_{\lambda}\left(\frac{1}{1-\lambda}\right)}{1-\lambda}\right)
$$

where $R^{*}=p^{*}\left(1-F\left(p^{*}\right)\right.$.

Notice that we have to assume existence and uniqueness of $p^{*}$ separately under $\lambda^{*}$ regularity, since Lemma 2 is no longer available. Similarly, $\lambda^{*}$-regularity does not imply Myerson's regularity.

\section{$5 \quad \lambda$-Antiregularity and Hyperregularity}

\section{$5.1 \lambda$-Antiregularity}

This section explores which of the results of the previous sections have counterparts in the $\lambda$-antiregular case in which the function $v_{\lambda}$ is decreasing instead of increasing. For $\lambda_{1}<\lambda_{2}, \lambda_{2}$-antiregularity implies $\lambda_{1}$-antiregularity. This direction of implications is opposite to the one in the $\lambda$-regular case. 0-antiregularity (decreasing failure rate) implies (-1)-antiregularity (decreasing density), while 0-regularity (increasing failure rate) implies 1-regularity (Myerson regularity). $\lambda_{1}$-antiregularity and $\lambda_{2}$-regularity can only hold simultaneously if $\lambda_{1} \leq \lambda_{2}$. If a distribution is simultaneously (-1)-antiregular and 1-regular, it has sufficiently heavy tails in the sense of a decreasing density, and not too heavy tails in the sense of Myerson regularity. Therefore, in many cases, assuming a decreasing density generates bounds in the opposite direction to those implied by $\lambda$-regularity.

As antiregularity only guarantees that tails are sufficiently heavy, it cannot guarantee integrability as in Proposition 2 (i), or existence and uniqueness of optimal reserve prices as in Lemma 2. We thus assume for the remainder of this section that 
distributions are 1-regular, and possess a finite expected value and a unique optimal reserve price. We next go through the results from the $\lambda$-regular case step by step, highlighting what happens when replacing $\lambda$-regularity by $\lambda$-antiregularity. In all cases, the proofs follow directly from those for the $\lambda$-regular case.

- Lemma 1 does not rely on $\lambda$-regularity and continues to hold. The boundary cases of Definition 1 are both $\lambda$-regular and $\lambda$-antiregular.

- The equivalences in Proposition 1 continue to hold with the following modifications: $v_{\lambda}$ being decreasing is equivalent to $r_{\lambda}$ being decreasing, the expression in part (iii) being convex, and $G_{1, \lambda}^{-1}(F(x))$ being concave.

- Conversely to Proposition 2 (ii), increasing convex transformations of a random variable with a $\lambda$-antiregular distribution have a $\lambda$-antiregular distribution.

- The five lower bounds in Lemma 3, Corollary 1 and Lemma 4 turn into upper bounds under antiregularity. The same is true for the analogous generalization of Corollary 4.

- The upper bound in Proposition 3 turns into a lower bound. Proposition 4 does not have a direct converse in the antiregular case since the estimates are not sharp.

- The sequence defined in Lemma 5 becomes decreasing and convex. Accordingly, the lower bound on the ratio of second and first order statistics in Corollary 2 is reversed. The lower bound on expected revenues in optimal auctions is not reversed however.

- The chain of inequalities in Lemma 6 is reversed and the lower bound in Corollary 3 becomes an upper bound.

\subsection{Hyperregularity}

Kleinberg and Yuan (2013) propose an alternative strengthening of Myerson's regularity which they call hyperregularity. They assume that, in addition to $F$ being 
1-regular, the function $v_{1}(x) / x$ is increasing, or, equivalently the rate

$$
x \cdot r_{0}(x)=\frac{x f(x)}{1-F(x)}
$$

is increasing. ${ }^{31}$ This monotonicity condition is well-known in the revenue management literature. There, it was introduced by Lariviere and Porteus (2001) under the name increasing generalized failure rate condition (IGFR) as a sufficient condition for unimodality of $x(1-F(x))$. We refer to Banciu and Mirchandani (2013) for a recent contribution with many references. Lariviere (2006) provides a summary of structural results about IGFR distributions, showing, for example, that the IGFR property is equivalent to the distribution of $\log (X)$ satisfying the classical IFR condition.

Ziya et al. (2004) provide a comparison between IGFR and Myerson regularity from the perspective of revenue management, concluding that, in general, neither condition is to be preferred over the other. ${ }^{32}$ IGFR is weaker than Myerson's regularity in the sense that it holds for distributions with arbitrarily heavy polynomial tails. Yet it is more restrictive in the sense that it may disappear under horizontal shifts of the distribution. For example, consider the parametric class of distributions $F(x)=1-(x+a)^{-b}$ with support $(1-a, \infty)$ where $a<1$ and $b>0$. We have $v_{\lambda}(x)=\left(\lambda-\frac{1}{b}\right) x+\frac{a}{b}$. $F$ is thus $\lambda$-regular for all $a$ and all $b \geq 1 / \lambda$. In particular, $F$ is Myerson regular for $b \geq 1$. The location parameter $a$ does not matter, but the heaviness of tails, parametrized by $b$, does. In contrast, whether the distributions are IGFR, i.e., whether $v_{1}(x) / x$ is increasing or decreasing, depends only on the sign of the location parameter $a . F$ is IGFR whenever $a \geq 0$, i.e., when the lower end of the support lies between 0 and $1 .^{33}$

Kleinberg and Yuan (2013) show that assuming hyperregularity has some attractive

\footnotetext{
${ }^{31}$ Neither of these assumption ensures integrability. To this end, Kleinberg and Yuan (2013) introduce the third assumption of $c$-boundedness which requires that the result of our Proposition 3 holds for some constant $c$, i.e., $E[X] \leq c p^{*}\left(1-F\left(p^{*}\right)\right)$.

${ }^{32}$ Ziya et al. (2004) also point out earlier papers from this literature which had "reinvented" Myerson regularity in the formulations (ii) and (iii) from Proposition 1, Bitran and Mondschein (1997) and Cachon and Lariviere (2001).

${ }^{33}$ These considerations illustrate the special role of the equal revenue distribution $a=0, b=1$, which is the only distribution at the boundary of both IGFR and Myerson's regularity. It is also a boundary case of the third related assumption discussed by Ziya et al. (2004), concavity of $x(1-F(x))$.
} 
implications in auction theory, see their Section 5. Yet, as shown in Section 3.2 above, their main argument for the unavoidability of assuming hyperregularity does not apply to distributions which are $\lambda$-regular for some $\lambda<1$. Assuming $\lambda$-regularity thus has the potential of replacing their three assumptions (regularity, IGFR and $c$-boundedness) by a single one.

\section{$6 \quad \lambda$-Regularity in Quantity Space}

Bulow and Roberts (1989) show that Myerson regularity becomes a classical concavity condition on a monopolist's revenue if the price-setting problem studied so far is transformed into a problem of choosing quantities. This section provides a brief and informal discussion of what $\lambda$-regularity means under this transformation. Let us interpret the function $1-F(p)$ as a demand curve and consider a monopolistic seller who sets price $p$. The seller's revenue $r(p)$, consumer surplus $c(p)$ and social welfare $s(p)$ (the sum of the former two) can then be written, respectively, as

$$
r(p)=p(1-F(p)), \quad c(p)=\int_{p}^{\infty}(x-p) f(x) d x=\int_{p}^{\infty} 1-F(x) d x .
$$

and

$$
s(p)=p(1-F(p))+\int_{p}^{\infty} 1-F(x) d x .
$$

Following Bulow and Roberts (1989), let us substitute $q=1-F(p)$ and interpret $1-F(p)$ as the quantity $q \in[0,1]$ sold when the unit price is $p$. Thus, we can write the price per unit when selling the quantity $q$ as $P(q)=F^{-1}(1-q)$. Under this transformation, revenue $R(q)$, consumer surplus $C(q)$ and social welfare $S(q)$ can be written as

$$
R(q)=q P(q), \quad C(q)=-\int_{0}^{q} y P^{\prime}(y) d y \quad \text { and } \quad S(q)=\int_{0}^{q} P(y) d y
$$

As $P$ is decreasing, social welfare $S$ is always concave in quantity. The generalized increasing virtual valuations formulation of $\lambda$-regularity turns into the condition that

$$
h_{\lambda}(q)=\lambda P(q)+q P^{\prime}(q)
$$


is decreasing in $q \cdot{ }^{34}$ For $\lambda=1$, we recover the result that $R^{\prime}(q)=h_{1}(q)$ is decreasing, implying that revenue is concave in $q$. For $\lambda=0$, the condition turns into $C^{\prime}(q)=$ $-h_{0}(q)$ being increasing. The monotone hazard rate assumption thus implies that consumer surplus is convex in quantity. Due to the concavity of $S$ and the relation $R(q)=S(q)-C(q)$, this is a sufficient condition for concavity of $R$. For general $\lambda<1$, we can write

$$
h_{\lambda}(q)=R^{\prime}(q)-(1-\lambda) S^{\prime}(q) .
$$

$\lambda$-regularity thus turns into a condition that revenue is sufficiently concave, requiring that revenue remains concave if social welfare times a positive, $\lambda$-dependent constant is subtracted.

\section{Conclusion}

Traditionally, properties like Myerson regularity and the monotone hazard rate condition were viewed as qualitative features that a distribution either fulfills or does not fulfill. Interpreting the smallest $\lambda$ for which a distribution is $\lambda$-regular as an index of regularity, allows for a more quantitative perspective. $\lambda$-regularity replaces the question whether a distribution is Myerson regular. It focuses instead on how Myerson regular a distribution is. Section 3 demonstrates that with regard to the performance of auctions and sales mechanisms, the answer often depends explicitly on the value of $\lambda$. Moreover, the results are sharp at boundary cases of $\lambda$-regularity. In a sense, $\lambda$-regularity is an almost naïve strengthening of Myerson regularity, replacing weak monotonicity by monotonicity with a lower bound on the slope. Yet, the concept has deep connections to the literatures on stochastic orders and on generalized concavity. In Section 3.1, we provided several pointers to other applications in which $\lambda$-regularity helps gaining a better understanding of how results depend on the regularity of distributions. We are convinced that there are many more contexts in which the concept will prove fruitful.

\footnotetext{
${ }^{34}$ Monotonicity of generalized failure rates turns into the equivalent condition that $q^{1+\lambda} P^{\prime}(q)$ is decreasing.
} 


\section{A Proofs}

We begin with an elementary calculation that is used in several of our proofs:

Lemma 7. Let $a \geq 0$ and $b>0$ and $\lambda<1$ with $\lambda \neq 0$ be three real numbers. When $\lambda<0$ assume in addition $a<-\frac{1}{\lambda}$. Then it holds that

$$
\int_{0}^{\infty} \Gamma_{\lambda}(a+b x) d x=\frac{\Gamma_{\lambda}(a)^{1-\lambda}}{(1-\lambda) b}
$$

Proof of Lemma \%. For $\lambda \in(0,1)$, the claim follows from

$$
\int_{0}^{\infty} \Gamma_{\lambda}(a+b x) d x=\frac{1}{b} \int_{a}^{\infty}(1+\lambda x)^{-\frac{1}{\lambda}} d x=\frac{1}{\lambda b} \int_{1+\lambda a}^{\infty} x^{-\frac{1}{\lambda}} d x=\frac{(1+\lambda a)^{-\frac{1-\lambda}{\lambda}}}{(1-\lambda) b} .
$$

For $\lambda<0$, we obtain the same result from the following modified argument:

$$
\int_{0}^{\infty} \Gamma_{\lambda}(a+b x) d x=\frac{1}{b} \int_{a}^{-\frac{1}{\lambda}}(1+\lambda x)^{-\frac{1}{\lambda}} d x=\frac{-1}{\lambda b} \int_{0}^{1+\lambda a} x^{-\frac{1}{\lambda}} d x=\frac{(1+\lambda a)^{-\frac{1-\lambda}{\lambda}}}{(1-\lambda) b}
$$

Proof of Lemma 1. We define

$$
H_{\lambda}(x)=\frac{(1-F(x))^{-\lambda}-1}{\lambda} .
$$

Since this expression satisfies $H_{\lambda}(0)=0$ and $H_{\lambda}^{\prime}(x)=r_{\lambda}(x)$, this definition is consistent with the integral for $H_{\lambda}$ given in the lemma. Applying $\Gamma_{\lambda}$ on both sides of $(2)$ gives $\left.1-F(x)=\Gamma_{\lambda}\left(H_{\lambda}(x)\right)\right)$. Finally, notice that $H_{\lambda}$ takes values in $\left[0,-\frac{1}{\lambda}\right]$ for $\lambda<0$ and in $[0, \infty)$ for $\lambda>0$, thus falling into the regions where $\Gamma_{\lambda}$ is non-zero.

Proof of Proposition 1. Equivalence of (i-iii) follows from the fact that all three claims correspond to the first order condition

$$
(1+\lambda) f(x)^{2}+(1-F(x)) f^{\prime}(x) \geq 0
$$


for all $x \in(\alpha, \omega)$. Equivalence of (iii) and (iv) follows from the fact that ${ }^{35}$

$$
G_{1, \lambda}^{-1}(F(x))=\frac{(1-F(x))^{-\lambda}-1}{\lambda} .
$$

which coincides with $H_{\lambda}$ by $(2)$.

Proof of Proposition 2. (i) is well-known for the boundary cases $F_{\mu, \lambda}$ and $G_{\rho, \lambda}$ which are simply rescaled Pareto distributions. The proof for general $F$ proceeds in two steps: We first show that $\lambda$-regularity with $\lambda<1$ implies existence of a finite mean $\mu$. Existence of higher moments is then deduced from a comparison with $F_{\mu, \lambda}$. Assume $F$ is not a boundary case so that $H_{\lambda}$ is not globally linear. Since $H_{\lambda}$ is convex and increasing with $H_{\lambda}(0)=0$, we can find a constant $\rho>0$ such that $\rho x$ and $H$ intersect exactly twice on $\mathbb{R}^{+}$, in 0 and at some $s>0$. By convexity, we have $H(x) \geq \rho x$ for $x \geq s$. We can thus bound the mean of $F$ by

$\mu=\int_{0}^{\infty} 1-F(x) d x=\int_{0}^{\infty} \Gamma_{\lambda}\left(H_{\lambda}(x)\right) d x \leq s+\int_{s}^{\infty} \Gamma_{\lambda}(\rho x) d x \leq s+\frac{1}{\rho(1-\lambda)}<\infty$.

where the final summand on the right hand side is the mean of $G_{\rho, \lambda}$. Notice next ${ }^{36}$ that the convex function $H_{\lambda}$ and the corresponding (linear) function $H_{\mu, \lambda}(x)=$ $\frac{x}{\mu(1-\lambda)}$ of $F_{\mu, \lambda}$ have at most one intersection at a positive $x$ where $H_{\lambda}$ intersects $H_{\mu, \lambda}$ from below. Accordingly, there exists $x_{0}$ such that $1-F(x) \geq 1-F_{\mu, \lambda}(x)$ for $x \leq x_{0}$ while the converse inequality holds for $x \geq x_{0}$. Combining this observation with

$$
\int_{0}^{\infty} 1-F(x) d x-\int_{0}^{\infty} 1-F_{\mu, \lambda}(x) d x=0
$$

shows that

$$
\int_{0}^{\infty} \varphi(x)(1-F(x)) d x-\int_{0}^{\infty} \varphi(x)\left(1-F_{\mu, \lambda}(x)\right) d x \leq 0
$$

for any increasing function $\varphi(x)$ by Lemma 7.1 of Chapter 4 in Barlow and Proschan (1981). To complete the proof of (i), it suffices to note that choosing $\varphi(x)=p x^{p-1}$ turns the latter inequality into a bound between the $p^{\text {th }}$ moments of $F$ and $F_{\mu, \lambda}$

\footnotetext{
${ }^{35}$ Notice that the choice of $\rho=1$ is arbitrary here. Dominance of $G_{1, \lambda}$ in the convex-transform order is equivalent to dominance of $G_{\rho, \lambda}$ for any $\rho>0$.

${ }^{36}$ The remainder of the proof of (i) is adapted from Theorem 4.8 and Corollary 4.9 in Barlow and Proschan (1965).
} 
since

$$
E\left[X^{p}\right]=\int_{0}^{\infty} p x^{p-1}(1-F(x)) d x .
$$

Existence of moments for $F_{\mu, \lambda}$ thus implies existence of moments for $F$. It remains to prove part (ii) of the proposition. Since $h$ is strictly increasing and concave, its inverse $h^{-1}$ exists and is convex. The cumulative distribution function of $h(X)$ is given by $F_{h}(x)=F\left(h^{-1}(x)\right)$. By Proposition $1, \lambda$-regularity of $F$ is equivalent to convexity of $H_{\lambda}(x)=G_{1, \lambda}(F(x))$. Since the composition of two convex functions is convex, we find that $G_{1, \lambda}\left(F_{h}(x)\right)=H_{\lambda}\left(h^{-1}(x)\right)$ is convex, implying $\lambda$-regularity of $F_{h}$.

Proof of Lemma 2. Since $v_{\lambda}$ is increasing for some $\lambda<1, v_{1}(x)=(1-\lambda) x+v_{\lambda}(x)$ is strictly increasing over $(\alpha, \omega)$, implying that $v_{1}$ has at most one zero. By Proposition 2, $F$ possesses a finite mean and thus

$$
E\left[v_{1}(x)\right]=\int_{0}^{\infty} x f(x) d x-\int_{0}^{\infty} 1-F(x) d x=0,
$$

so that $v_{1}$ must have at least one zero which lies in the interior of the support.

Proof of Lemma 3. Since $H_{\lambda}$ is convex, we have $H_{\lambda}(\mu) \leq E\left[H_{\lambda}(X)\right]$ by Jensen's inequality. We claim that $E\left[H_{\lambda}(X)\right]=\frac{1}{1-\lambda}$. Using that $\Gamma_{\lambda}$ is decreasing then yields

$$
1-F(\mu)=\Gamma_{\lambda}\left(H_{\lambda}(\mu)\right) \geq \Gamma_{\lambda}\left(E\left[H_{\lambda}(X)\right]\right)=\Gamma_{\lambda}\left(\frac{1}{1-\lambda}\right)
$$

which is the first inequality. To see the claim, we write

$$
\begin{aligned}
E\left[H_{\lambda}(X)\right] & =\int_{0}^{\infty} H_{\lambda}(x) f(x) d x=\int_{0}^{\infty} \int_{0}^{x} r_{\lambda}(y) f(x) d y d x \\
& =\int_{0}^{\infty} r_{\lambda}(y) \int_{y}^{\infty} f(x) d x d y=\int_{0}^{\infty}(1-F(y))^{-\lambda} f(y) d y
\end{aligned}
$$

where the last step uses the definition of $r_{\lambda}$. The substitution $u=F(y)$ then gives

$$
E\left[H_{\lambda}(X)\right]=\int_{0}^{1}(1-u)^{-\lambda} d u=\frac{1}{1-\lambda} .
$$


For the second inequality, we rewrite the condition $v_{1}\left(p^{*}\right)=0$ into

$$
p^{*} r_{\lambda}\left(p^{*}\right)=\left(1-F\left(p^{*}\right)\right)^{-\lambda}=\Gamma_{\lambda}\left(H_{\lambda}\left(p^{*}\right)\right)^{-\lambda}=1+\lambda H_{\lambda}\left(p^{*}\right),
$$

using the definitions of $r_{\lambda}$ and $\Gamma_{\lambda}$. Since $H_{\lambda}$ is convex with $H_{\lambda}^{\prime}=r_{\lambda}$ and $H_{\lambda}(0)=0$, we obtain by (3) that

$$
H_{\lambda}\left(p^{*}\right) \leq p^{*} r_{\lambda}\left(p^{*}\right)=1+\lambda H_{\lambda}\left(p^{*}\right)
$$

Solving this bound for $H_{\lambda}\left(p^{*}\right)$ yields $H_{\lambda}\left(p^{*}\right) \leq \frac{1}{1-\lambda}$. Applying the decreasing function $\Gamma_{\lambda}$ to both sides of this inequality completes the proof since $1-F\left(p^{*}\right)=\Gamma_{\lambda}\left(H_{\lambda}\left(p^{*}\right)\right)$.

Proof of Corollary 1. We give a combined proof of both assertions. By Lemma 3, we have $H_{\lambda}(0)=0$ and $H_{\lambda}(z) \leq \frac{1}{1-\lambda}$ for $z \in\left\{\mu, p^{*}\right\}$. Convexity of $H_{\lambda}$ thus implies

$$
H_{\lambda}(x) \leq \frac{x}{z(1-\lambda)}
$$

for $x \in[0, z]$. Applying the decreasing function $\Gamma_{\lambda}$ to this inequality yields

$$
1-F(x)=\Gamma_{\lambda}\left(H_{\lambda}(x)\right) \geq \Gamma_{\lambda}\left(\frac{x}{z(1-\lambda)}\right)=1-F_{z, \lambda}(x)
$$

by Definition 1.

Proof of Lemma 4. We need to show an upper bound on $\Gamma_{\lambda}\left(H_{\lambda}\left(p^{*} \Gamma_{\lambda}\left(H_{\lambda}\left(p^{*}\right)\right)\right)\right)$. Since $H_{\lambda}$ is convex with $H_{\lambda}(0)=0$ and since $\Gamma_{\lambda}\left(H_{\lambda}\left(p^{*}\right)\right)=1-F\left(p^{*}\right) \in(0,1)$, we obtain

$$
H_{\lambda}\left(p^{*} \Gamma_{\lambda}\left(H_{\lambda}\left(p^{*}\right)\right)\right) \leq H_{\lambda}\left(p^{*}\right) \cdot \Gamma_{\lambda}\left(H_{\lambda}\left(p^{*}\right)\right)=\Phi\left(H_{\lambda}\left(p^{*}\right)\right) .
$$

where $\Phi(x)=x \cdot \Gamma_{\lambda}(x)$. Since $\Phi$ has derivative

$$
\Phi^{\prime}(x)=\Gamma_{\lambda}(x)\left(1-\frac{x}{1+\lambda x}\right)
$$


$\Phi$ is increasing for $x \leq(1-\lambda)^{-1}$ and decreasing for $x \geq(1-\lambda)^{-1}$. We obtain ${ }^{37}$

$$
H_{\lambda}\left(p^{*} \Gamma_{\lambda}\left(H_{\lambda}\left(p^{*}\right)\right)\right) \leq \Phi\left(H_{\lambda}\left(p^{*}\right)\right) \leq \Phi\left(\frac{1}{1-\lambda}\right)=\frac{1}{1-\lambda} \Gamma_{\lambda}\left(\frac{1}{1-\lambda}\right)
$$

Applying the decreasing function $\Gamma_{\lambda}$ on both sides of the inequality completes the proof.

Proof of Proposition 3. Since $H_{\lambda}$ is convex with $H_{\lambda}^{\prime}=r_{\lambda}$, we have $H_{\lambda}(x) \geq H_{\lambda}\left(p^{*}\right)+$ $r_{\lambda}\left(p^{*}\right)\left(x-p^{*}\right)$ and thus

$$
\mu=\int_{0}^{\infty} 1-F(x) d x=\int_{0}^{\infty} \Gamma_{\lambda}\left(H_{\lambda}(x)\right) d x \leq \int_{0}^{\infty} \Gamma_{\lambda}\left(H_{\lambda}\left(p^{*}\right)+r_{\lambda}\left(p^{*}\right)\left(x-p^{*}\right)\right) d x .
$$

The constants $a=H_{\lambda}\left(p^{*}\right)-p^{*} r_{\lambda}\left(p^{*}\right)$ and $b=r_{\lambda}\left(p^{*}\right)$ are non-negative by (4). For $\lambda<0$, we further have $a \leq H_{\lambda}\left(p^{*}\right) \leq-\frac{1}{\lambda}$ as noted in the proof of Lemma 1 . Applying Lemma 7 with these constants $a$ and $b$ thus yields

$$
\mu \leq \frac{\Gamma_{\lambda}\left(H_{\lambda}\left(p^{*}\right)-p^{*} r_{\lambda}\left(p^{*}\right)\right)^{1-\lambda}}{(1-\lambda) r_{\lambda}\left(p^{*}\right)}
$$

By (3), we have $p^{*} r_{\lambda}\left(p^{*}\right)=1+\lambda H_{\lambda}\left(p^{*}\right)$ and thus

$$
\Gamma_{\lambda}\left(H_{\lambda}\left(p^{*}\right)-p^{*} r_{\lambda}\left(p^{*}\right)\right)=\Gamma_{\lambda}\left((1-\lambda) H_{\lambda}\left(p^{*}\right)-1\right)=(1-\lambda)^{-\frac{1}{\lambda}} \Gamma_{\lambda}\left(H_{\lambda}\left(p^{*}\right)\right)
$$

where the final step uses the definition of $\Gamma_{\lambda}$. (5) can thus be written as

$$
\mu \leq \frac{(1-\lambda)^{\frac{\lambda-1}{\lambda}}}{(1-\lambda)} \cdot \frac{\Gamma_{\lambda}\left(H_{\lambda}\left(p^{*}\right)\right)^{1-\lambda}}{r_{\lambda}\left(p^{*}\right)}=(1-\lambda)^{-\frac{1}{\lambda}} \cdot p^{*} \Gamma_{\lambda}\left(H_{\lambda}\left(p^{*}\right)\right)
$$

where the last step follows from (3). As $\Gamma_{\lambda}\left(H_{\lambda}\left(p^{*}\right)\right)=1-F\left(p^{*}\right)$, the proof is complete.

Proof of Proposition 4. The first bound follows directly from Proposition 3 and $E\left[X 1_{\{X \geq p\}}\right] \leq \mu$. For the second, notice first that by partial integration

$$
E\left[X 1_{\{X \geq p\}}\right]=\int_{p}^{\infty} x f(x) d x=p(1-F(p))+\int_{p}^{\infty} 1-F(x) d x .
$$

\footnotetext{
${ }^{37}$ This bound is sharp at the boundary cases of $\lambda$-regularity which satisfy $H_{\lambda}\left(p^{*}\right)=\frac{1}{1-\lambda}$.
} 
It thus suffices to bound the second summand by $(1-\lambda)^{-1} p(1-F(p))$. By convexity of $H_{\lambda}$, we obtain

$$
\begin{aligned}
\int_{p}^{\infty} 1-F(x) d x & =\int_{p}^{\infty} \Gamma_{\lambda}\left(H_{\lambda}(x)\right) d x \leq \int_{p}^{\infty} \Gamma_{\lambda}\left(H_{\lambda}(p)+r_{\lambda}(p)(x-p)\right) d x \\
& =\int_{0}^{\infty} \Gamma_{\lambda}\left(H_{\lambda}(p)+r_{\lambda}(p) y\right) d y=\frac{\Gamma_{\lambda}\left(H_{\lambda}(p)\right)^{1-\lambda}}{(1-\lambda) r_{\lambda}(p)}
\end{aligned}
$$

where the last step uses Lemma 7 with constants $a=H_{\lambda}(p)$ and $b=r_{\lambda}(p) . a$ and $b$ are non-negative, and, as argued in the proof of Lemma 1, we have $a \leq-\frac{1}{\lambda}$ for $\lambda<0$. Arguing as in (3), we conclude from $p \geq p^{*}$ that $v_{1}(p) \geq 0$ and thus $\Gamma_{\lambda}\left(H_{\lambda}(p)\right)^{-\lambda} \leq$ $p r_{\lambda}(p)$. Combining this estimate with (6) yields the desired inequality

$$
\int_{p}^{\infty} 1-F(x) d x \leq \frac{p \Gamma_{\lambda}\left(H_{\lambda}(p)\right)}{1-\lambda}=\frac{p(1-F(p))}{1-\lambda} .
$$

Proof of Lemma 5. For any increasing function $h$ with $E\left[h\left(X_{1}\right)\right]<\infty$, the sequence $E\left[h\left(X_{1: n}\right)\right]$ is increasing and concave in $n$, since it is the sequence of first order statistics of the transformed random variables $h\left(X_{i}\right)$, and since sequences of first order statistics are always increasing and concave, see Szech (2011), Lemma 1. The claim of the lemma follows from this observation by choosing the increasing function as $h(x)=v_{\lambda}(x)$. To see this, notice first that $a_{1}=E\left[v_{\lambda}\left(X_{1: 1}\right)\right]=(1-\lambda) E[X]$. Moreover, denote by $F_{1: n}(x)=F(x)^{n}, f_{1: n}(x)=n F(x)^{n-1} f(x)$ the distribution function and density of $X_{1: n}$ and by $F_{2: n}(x)=F_{1: n}(x)+n(1-F(x)) F(x)^{n-1}$ the distribution function of $X_{2: n} \cdot{ }^{38}$ Then we obtain

$$
\begin{aligned}
a_{n} & =E\left[v_{\lambda}\left(X_{1: n}\right)\right]=\lambda E\left[X_{1: n}\right]-\int_{0}^{\infty} \frac{(1-F(x))}{f(x)} \cdot f_{1: n}(x) d x \\
& =\lambda E\left[X_{1: n}\right]+\int_{0}^{\infty} F_{2: n}(x)-F_{1: n}(x) d x=\lambda E\left[X_{1: n}\right]-\left(E\left[X_{1: n}-X_{2: n}\right]\right)
\end{aligned}
$$

for $n \geq 2$ which completes the proof.

Proof of Corollary 2. The first inequality $R_{n}^{*} \geq E\left[X_{2: n}\right]$ simply restates that revenueoptimal auctions dominate auctions without reserve in terms of revenue. The second

\footnotetext{
${ }^{38}$ For background see David and Nagaraja (2003).
} 
inequality is a rearrangement of the implication $a_{n} \geq a_{1}$ of Lemma 5 .

Proof of Lemma 6 . We begin with the second inequality between the mean and the minimum. Notice first that for any $\theta>-1$ we have

$$
\int_{0}^{\infty}\left(1-F(x)^{\theta} f(x) d x=\int_{0}^{1}(1-u)^{\theta} d u=\frac{1}{1+\theta} .\right.
$$

Therefore, the function

$$
g(x)=\left(\frac{2-\lambda}{1-\lambda}(1-F(x))-1\right)(1-F(x))^{-\lambda} f(x)
$$

satisfies $\int_{0}^{\infty} g(x) d x=0$. Moreover, by monotonicity and positivity of $1-F, g$ switches signs exactly once, from positive to negative. By Lemma 7.1 of Chapter 4 in Barlow and Proschan (1981), we thus have for any decreasing function $h$ that

$$
0 \leq \int_{0}^{\infty} h(x) g(x) d x
$$

Choosing the decreasing function $h$ as $h(x)=\frac{1}{r_{\lambda}(x)}$ yields

$$
0 \leq \frac{2-\lambda}{1-\lambda} \int_{0}^{\infty}(1-F(x))^{2} d x-\int_{0}^{\infty} 1-F(x) d x=\frac{2-\lambda}{1-\lambda} E\left[X_{2: 2}\right]-E\left[X_{1}\right] .
$$

The inequality for the maximum follows from the one for the minimum via

$$
E\left[X_{1: 2}\right]=2 E\left[X_{1}\right]-E\left[X_{2: 2}\right] \leq\left(2-\frac{1-\lambda}{2-\lambda}\right) E\left[X_{1}\right]=\frac{3-\lambda}{2-\lambda} E\left[X_{1}\right]
$$

Proof of Corollary 3. Notice that the distribution function of $X_{n: n}$ is $F_{n: n}(x)=1-$ $(1-F(x))^{n}$ and thus

$$
E\left[X_{n: n}\right]=\int_{0}^{\infty}(1-F(x))^{n} d x .
$$

The proof of the corollary is analogous to the bound for $X_{2: 2}$ in Lemma 6 except that we choose the function $g$ as

$$
g(x)=\left(\frac{n-\lambda}{1-\lambda}(1-F(x))^{n-1}-1\right)(1-F(x))^{-\lambda} f(x)
$$


Proof of Proposition 5. Equivalence of (i) and (ii) follows since the first order condition associated with (i),

$$
0 \leq \frac{H_{\lambda}^{\prime}(x) x-H_{\lambda}(x)}{x^{2}}=\frac{r_{\lambda}(x) x-H_{\lambda}(x)}{x^{2}}
$$

is equivalent to (ii). For $y \leq x$, we can choose $\theta=y / x \in[0,1]$. (i) and (iii) are then both the same as $H_{\lambda}(y) / y \leq H_{\lambda}(x) / x$ for all $y \leq x$.

Proof of Corollary 4. The first inequality in the corollary comes from Lemma 3 in the $\lambda$-regular case. The only consequence of $\lambda$-regularity used in its proof is $H_{\lambda}\left(p^{*}\right) \leq$ $p^{*} r_{\lambda}\left(p^{*}\right)$. This holds under $\lambda^{*}$-regularity by Proposition 5 (ii). The second inequality is proved in Corollary 1. The proof is based on the first inequality and the fact that $H_{\lambda}(x) \leq \frac{x}{z} H_{\lambda}(z)$ for $x \leq z$, the definition of $\lambda^{*}$-regularity. The third inequality is from Lemma 4 . The only application of $\lambda$-regularity in the proof follows from part (iii) of Proposition 5 with $\theta=\Gamma_{\lambda}\left(H_{\lambda}\left(p^{*}\right)\right)$ and $x=p^{*}$.

\section{References}

Gagan Aggarwal, Gagan Goel, and Aranyak Mehta. Efficiency of (revenue-)optimal mechanisms. In Proceedings of the 10th ACM conference on Electronic commerce, pages 235-242. ACM, 2009.

Simon P. Anderson and Régis Renault. Efficiency and surplus bounds in Cournot competition. Journal of Economic Theory, 113(2):253-264, 2003.

Pablo D. Azar, Constantinos Daskalakis, Silvio Micali, and S. Matthew Weinberg. Optimal and efficient parametric auctions. In Proceedings of the 24th Annual ACM-SIAM Symposium on Discrete Algorithms, pages 596-604. SIAM, 2013.

Pablo D. Azar, Robert Kleinberg, and S. Matthew Weinberg. Prophet inequalities with limited information. In Proceedings of the 25th Annual ACM-SIAM Symposium on Discrete Algorithms, pages 1358-1377. SIAM, 2014.

Mark Bagnoli and Ted Bergstrom. Log-concave probability and its applications. Economic Theory, 26(2):445-469, 2005. 
Tamás L. Balogh and Christian Ewerhart. On the origin of $r$-concavity and related concepts. Technical Report 187, Department of Economics, University of Zurich, 2014.

Mihai Banciu and Prakash Mirchandani. New results concerning probability distributions with increasing generalized failure rates. Operations Research, 61(4):925-931, 2013.

Richard E. Barlow and Frank Proschan. Mathematical Theory of Reliability. Wiley, 1965.

Richard E. Barlow and Frank Proschan. Inequalities for linear combinations of order statistics from restricted families. Annals of Mathematical Statistics, 37(6):1574-1592, 1966 .

Richard E. Barlow and Frank Proschan. Statistical Theory of Reliability and Life Testing. Holt, Rinehart \& Winston, 2nd edition, 1981.

Gabriel R. Bitran and Susana V. Mondschein. Periodic pricing of seasonal products in retailing. Management Science, 43(1):64-79, 1997.

Jeremy Bulow and John Roberts. The simple economics of optimal auctions. Journal of Political Economy, 97(5):1060-1090, 1989.

Gérard P. Cachon and Martin A. Lariviere. Contracting to assure supply: How to share demand forecasts in a supply chain. Management Science, 47(5):629-646, 2001.

Andrew Caplin and Barry Nalebuff. Aggregation and social choice: a mean voter theorem. Econometrica, 59(1):1-24, 1991a.

Andrew Caplin and Barry Nalebuff. Aggregation and imperfect competition: On the existence of equilibrium. Econometrica, 59(1):25-59, 1991b.

Luděk Cigler, Wolfgang Dvořák, Monika Henzinger, and Martin Starnberger. Limiting price discrimination when selling products with positive network externalities. In $\mathrm{Web}$ and Internet Economics, pages 44-57. Springer, 2014.

Richard Cole and Sravas Rao. Applications of $\alpha$-strongly regular distributions to Bayesian auctions. ArXiv preprint 1512.02285, December 2015.

Richard Cole and Tim Roughgarden. The sample complexity of revenue maximization. In Proceedings of the 46th Annual ACM Symposium on Theory of Computing, pages 243-252. ACM, 2014.

Constantinos Daskalakis and George Pierrakos. Simple, optimal and efficient auctions. In Internet and Network Economics, pages 109-121. Springer, 2011. 
Herbert A. David and Haikady N. Nagaraja. Order Statistics. John Wiley \& Sons, 3rd edition, 2003.

Peerapong Dhangwatnotai, Tim Roughgarden, and Qiqi Yan. Revenue maximization with a single sample. Games and Economic Behavior, 91:318-333, 2014.

David Donaldson and John A. Weymark. Ethically flexible Gini indices for income distributions in the continuum. Journal of Economic Theory, 29(2):353-358, 1983.

Christian Ewerhart. Regular type distributions in mechanism design and $\rho$-concavity. Economic Theory, 53(3):591-603, 2013.

Drew Fudenberg and Jean Tirole. Game Theory. MIT Press, 1991.

Jason D. Hartline. Mechanism Design and Approximation. Monograph in progress, available at http://jasonhartline.com/MDnA, 2014.

Jason D. Hartline, Vahab Mirrokni, and Mukund Sundararajan. Optimal marketing strategies over social networks. In Proceedings of the 17th International World Wide Web conference, pages 189-198. ACM, 2008.

Christian Kleiber and Samuel Kotz. Statistical Size Distributions in Economics and Actuarial Sciences. John Wiley \& Sons, 2003.

Robert Kleinberg and Yang Yuan. On the ratio of revenue to welfare in single-parameter mechanism design. In Proceedings of the 14th ACM conference on Electronic commerce, pages 589-602. ACM, 2013.

Martin A. Lariviere. A note on probability distributions with increasing generalized failure rates. Operations Research, 54(3):602-604, 2006.

Martin A. Lariviere and Evan L. Porteus. Selling to the newsvendor: An analysis of price-only contracts. Manufacturing \& Service Operations Management, 3(4):293-305, 2001.

Vlad Mares and Jeroen M. Swinkels. Near-optimality of second price mechanisms in a class of asymmetric auctions. Games and Economic Behavior, 72(1):218-241, 2011.

Vlad Mares and Jeroen M. Swinkels. On the analysis of asymmetric first price auctions. Journal of Economic Theory, 152(1):1-40, 2014.

Albert W. Marshall and Ingram Olkin. Life Distributions. Springer, 2007. 
R. Preston McAfee and John McMillan. Auctions and bidding. Journal of Economic Literature, 25(2):699-738, 1987.

Benny Moldovanu, Aner Sela, and Xianwen Shi. Contests for status. Journal of Political Economy, 115(2):338-363, 2007.

Benny Moldovanu, Aner Sela, and Xianwen Shi. Competing auctions with endogenous quantities. Journal of Economic Theory, 141(1):1-27, 2008.

Roger B. Myerson. Optimal auction design. Mathematics of Operations Research, 6(1): 58-73, 1981.

Tim Roughgarden. Approximately optimal mechanism design: Motivation, examples, and lessons learned. ACM SIGecom Exchanges, 13(2):4-20, 2015.

Moshe Shaked and J. George Shanthikumar. Stochastic Orders. Springer, 2007.

Mukund Sundararajan and Qiqi Yan. Robust mechanisms for risk-averse sellers. Games and Economic Behavior, forthcoming, 2015.

Nora Szech. Optimal advertising of auctions. Journal of Economic Theory, 146(6):2596$2607,2011$.

Willem R. van Zwet. Convex Transformations of Random Variables. Mathematisch Centrum Amsterdam, 1964.

E. Glen Weyl and Michal Fabinger. Pass-through as an economic tool: Principles of incidence under imperfect competition. Journal of Political Economy, 121(3):528-583, 2013.

Robert Wilson. Game-theoretic analyses of trading processes. In Truman F. Bewley, editor, Advances in Economic Theory, pages 33-70. Cambridge University Press, 1987.

Qiqi Yan. Prior-Independence: A New Lens for Mechanism Design. PhD thesis, Stanford University, 2012.

Serhan Ziya, Hayriye Ayhan, and Robert D. Foley. Relationships among three assumptions in revenue management. Operations Research, 52(5):804-809, 2004. 